\title{
LA ORDENACIÓN DEL TERRITORIO EN EL PROTECTORADO ESPAÑOL EN MARRUECOS
}

\author{
Dr. D. Abel Albet i Mas \\ Universidad Autónoma de Barcelona. España
}

PRESENTACIÓN*

A pesar de que con anterioridad existe alguna propuesta de intervención ordenada sobre el territorio marroquí, es a partir de los años 1940 cuando empieza una verdadera politica de planificación territorial que será introducida en el Protectorado incluso antes que en la Peninsula, donde la redacción e implementación de planes todavía no era una práctica habitual. Si bajo el franquismo la planificación económica muestra contradicciones considerables, la política de ordenación en Marruecos es un ejemplo de su carácter tecnocrático; dado que los trabajos hidráulicos son una verdadera obsesión del régimen, el acento es puesto en las obras para aumentar el regadio, en un momento en que, por evidentes razones ideológicas, las preferencias se decantan por las reformas técnicas más que las de tipo social o económico.

Es en este contexto que se redactan diversos planes sectoriales (obras hidrálicas, puertos, carreteras, ferrocarriles, agricultura y bosques), se traza una política urbanística para toda la Zona, y se marcan las líneas de lo que pretendía ser una política territorial amplia encuadrada en un plan territorial global y secuenciada en unos planes quinquenales. Este esfuerzo planificador se concreta en la obra de síntesis Acción de España en Marruecos que, para su epoca y circunstancias y a pesar de sus limitaciones y contradicciones internas, se muestra como un singular y excelente ejercicio de ordenación territorial.

\section{LAS OBRAS HIDRÁULICAS}

A pesar de que durante muchos años tanto los discursos de técnicos, políticos e ideólogos como la evidencia cotidiana coincidían en la urgencia de plantear la resolución de la problemática hidrológica como factor clave en el proceso de colonización del territorio

* El contenido de este texto se enmarca en el proyecto de investigación PB93-0551 financiado por la DGICyT del Ministerio de Educación y Ciencia y en un capitulo, escrito por el mismo autor, de la obra España en Marruecos (1912-1956). Discursos geográficos e intervención territorial Lleida: Milenio, 1999 coordinado por Joan Nogué y José Luis Villanova. 
marroqui,' no es hasta 1934 cuando, gracias a la iniciativa de una empresa privada, empiezan a quedar resueltas necesidades tan esenciales como el abastecimiento de agua potable y de electricidad de Ceuta, Tetuán, Larache o Tánger. ${ }^{2}$ Con anterioridad al establecimiento del Protectorado tan sólo existían algunas acequias locales destinadas a regadíos de pequeña escala mientras que entre 1912 y 1928 las únicas obras que se realizan son cierto número de captaciones de agua que debían contribuir parcialmente a cubrir las necesidades de la tropa.

La administración pública empieza a efectuar actuaciones significativas (aunque precarias) desde 1938, pero cuando a partir de 1940 se inician los trabajos sistemáticos para dotar a la Zona de un marco amplio de planificación territorial, es de remarcar la prioridad absoluta concedida al tema del agua (abastecimiento a los núcleos habitados, regadíos, producción eléctrica, etc.), justificada parcialmente ante la necesidad de asegurar las provisiones de alimentos a la población autóctona pero también debido a la verdadera "obsesión hidráulica" del régimen de Franco que entronca con una de las preocupaciones básicas del regeneracionismo español de principios de siglo. Además, la escasez de combustible en el contexto de la II Guerra Mundial provocó el aceleramiento en la construcción de algunas presas, contribuyendo a cubrir las necesidades de energía eléctrica (Gudín, 1962: p. 108).

Así pues, de entre los diversos planes sectoriales redactados, el de Obras Hidráulicas fue el primero, ${ }^{3}$ siendo presentado en 1942 . A pesar de la precariedad en los medios y de la escasez de datos disponibles el Plan contempla un estudio amplio y detallado para cada cuenca fluvial de la Zona del Protectorado, incluyendo un estudio sobre su orografia, pluviometría, caudales, aforos, etc. pero también sobre las posibilidades de aprovechamiento (riegos, producción eléctrica potencial, etc.), llegando a realizarse cálculos acerca de la productividad agraria potencial una vez convertidos en regadio un determinado número de campos todavía baldío dado que se incluyen presupuestos muy detallados acerca de cada obra y sus repercusiones en el territorio. A pesar de que el déficit agricola deberá cubrirse siempre desde la Península, el Plan propicia una situación de máxima autosuficiencia en los que respecta a la capacidad energética. ${ }^{4}$

Aquellos estudios sobre las potencialidades hídricas de la Zona se ofrecen en una visión parcial, para cada cuenca o valle, pero también en una perspectiva global, a la búsqueda de balances que aseguren soluciones óptimas. Las disparidades detectadas no auguran una fácil implementación de lo planificado:

\footnotetext{
"Las obras hidráulicas constituyen el núcleo revalorizador de la Zona" (Martorell, 1946b: p. 171).

Tal y como queda reflejado en el capítulo 13 de esta misma obra. Véase también Lambea (1942a: p. 1).

3 También la obra de síntesis de toda la planificación territorial marroquí Acción de España en Marruecos. La obra material (Alta Comisaría de España en Marruecos, 1948) concede una importancia preferente a este Plan, dedicándole un considerable número de páginas en sus primeros capítulos, remarcando el hecho de que se trata de un tema prioritario.

4 Además de los textos originales del redactor del Plan, el Ingeniero de Caminos, Canales y Puertos Daniel Lambea Palacios (especialmente Lambea, 1942a: pp. 5-6 y Lambea, 1942b: pp. 12-19), véase también los de Martorell (1946b: pp. 173-174) y González Quijano (1942).
} 
"La Zona se divide en dos regiones: la Oriental o seca y la Occidental o humeda. La divisoria entre ambas queda marcada por el rio Nekor. En la región húmeda tenemos agua en exceso mientras que en la seca nos falta. Podría creerse a primera vista que la solución está en llevarse el excedente de una región a la otra y compensar el déficit; las obras no son viables económicamente. La topografia no aporta grandes ayudas." (Lambea, 1942a: pp. 8-9)

no obstante, se es muy consciente de la trascendencia que la correcta y urgente explotación hidrológica tiene para la sociedad y la economía marroquí:

"Dejando aparte el buen refuerzo que representan las dos primeras llas riquezas mineras y pesqueras] toda la economia de Marruecos ha de girar alrededor de sus obras hidráulicas que en definitiva son las que han de conseguir la elevación del nivel medio de vida del pais, en el equilibrio de su balance económico." (Lambea, 1942b: p. 6)

No obstante la importancia y la urgencia reconocida a la cuestión hidráulica, el grado de cumplimiento de lo planificado será bastante reducido (Gudín, 1962: pp. 105-112) recogiendo, además, la práctica totalidad de las casuísticas típicas observadas en lo que respecta al (no) desarrollo de la política territorial en Marruecos: contradicciones en las iniciativas, desidia de la administración, corrupción de los gestores, falta de criterio, etc. Un ejemplo perfecto de ello es el caso del aprovechamiento del río Muluya (uno de los más caudalosos y regulares de la Zona) cuya cuenca forma un amplio valle que, desde principios de siglo, se intuye puede ser una óptima área de desarrollo agrícola: de manera reiterada se remarca que la presa en el Muluya y la canalización de sus aguas serán, sin lugar a dudas, la gran infraestructura que transformará el extremo oriental del Protectorado y que, por lo tanto, está destinada a ser exponente destacado y paradigmático de la labor y la forma de proceder de la intervención española.

Dado que la frontera con la zona francesa coincide con el curso del río durante un largo trecho de su recorrido, desde el mismo momento de la implantación del Protectorado se iniciaron conversaciones para llevar a cabo algún tipo de explotación conjunta: si el 1914 se redacta un proyecto de convenio, hasta 1926 no es posible un acuerdo que establece el reparto de costes y aprovechamientos en el que, contrariamente al objetivo de los españoles, los franceses sólo pretenden una simple derivación de aguas. Entre 1931 y 1947 los estudios técnicos previos están totalmente parados, primero por el escaso interés demostrado por el gobierno republicano español $y$, posteriormente, al verse los franceses involucrados en el conflicto bélico mundial. Finalmente, la construcción de la presa se inicia en 1951 finalizándose las obras en 1956, año de la independencia marroquí (Cámara, 1932: pp. 3, 13-14; Gudin, 1962: p. 110).

\section{LA ORDENACIÓN PORTUARIA}

El Plan de Ordenación Portuaria de la Zona del Protectorado se redacta en el año 1943 y se reconoce como el primer estudio de conjunto realizado de una manera seria y sistemá- 
tica (Alta Comisaria de España en Marruecos, 1948: p. 534). ${ }^{5}$ Tal y como sucede en otros planes referidos a la Zona, este incluye extensos trabajos previos que se justifican por la inexistencia de estadísticas y referencias anteriores pero que a la vez sirven de marco para el planteamiento y resolución de los problemas detectados. Asimismo, en esta ocasión es especialmente sensible la dificultad de considerar separadamente los puertos de Ceuta y Melilla (asi como el de Tánger) de los del resto de localidades del Protectorado. En esta línea de argumentación, se demuestra que en el momento de redactar el Plan, el tránsito de estos tres puertos concentra el $65 \%$ de las importaciones del Marruecos septentrional, de manera que suponen los únicos centros capaces para acoger la navegación de altura.

El Plan reconoce que los tres puertos existentes son suficientes para las necesidades y características de la región en relación al exterior. No obstante, no se opina lo mismo respecto de los puertos de cabotaje y la navegación que de ellos debería derivarse: para una adecuada distribución de las mercancías dentro del extenso perímetro marítimo de la Zona, la inexistencia de una red de pequeños puertos que respondan a las necesidades del tráfico local ${ }^{6}$ obliga a recurrir al transporte por carretera, con un indudable encarecimiento de los costes.

El documento del Plan presenta un detallado estudio de los puertos de Larache, Arcila, Rio Martín y Villa Sanjurjo así como las posibilidades de la Mar Chica en relación con el puerto de Melilla. Para cada uno de ellos se presentan planos detallados que especifican la situación presente y las obras proyectadas a la vez que se hace una amplia referencia a su historia, a la problemática que generan las corrientes, mareas, rios, lodos, olas u otros elementos de la naturaleza, así como otros aspectos económicos de la actividad portuaria (Ochoa, 1944: pp. 343-355). Como ocurre en otros planes, se trata de una aproximación rigurosa a pesar de la precariedad de datos y estadísticas (profundidades, vientos, etc.); el conocimiento de la situación se ha contrastado con un extenso trabajo de campo que a menudo se recoge en el mismo Plan en forma de opiniones procedentes de los propios habitantes y usuarios de los puertos. A pesar, pues, de que no se llegan a concretar esquemas precisos, el Plan es suficientemente detallado como para no tan sólo ordenar el litoral marroquí desde el punto de vista de los transportes marítimos sino de proponer soluciones concretas a problemáticas precisas de cada puerto: su integración urbana, su hinterland, su capacidad de atraque, etc. (Martorell, 1946b: p. 177).

Las propuestas plantean que Larache, Villa Sanjurjo y Río Martín sean considerados puertos comerciales y que se construyan las infraestructuras y equipamientos que les confieran esta calificación y nivel. Además de estos tres, el de Arcila debe ser considerado puerto de pesca; Puerto Capaz es calificado como puerto de refugio y Lau, Alcazarseguer y

5 En 1906 se aprobó el proyecto de puerto para Ceuta y en 1907 se iniciaban las obras del de Melilla pero en ambos casos se plantearon como infraestructuras para las respectivas ciudades y sin relación directa con el resto del territorio marroquí ni en el marco de ninguna lógica o jerarquía global (Gómez-Jordana, 1976: p. 22).

Entendiendo este tráfico local también el existente con los puertos de la Costa del Sol, ya que un buen tramo de costa rifeña y peninsular discurren en paralelo a una distancia media de tan sólo $150 \mathrm{kms}$., lo que debería propiciar unos flujos fáciles y directos entre ambas orillas del Mar de Alborán. 
Torres de Alcalá son simples embarcaderos. A pesar de que no existe ninguna propuesta acerca de Ceuta o Melilla, implícitamente ambos están presentes a lo largo de todo el documento ya que, como se ha dicho, suponen los núcleos imprescindibles de una jerarquía de puertos, sin los cuales el resto de la red pierde sentido.

Otra de las singularidades a destacar de este Plan es su carácter razonado, dialogado, abierto: de cada puerto, de cada problema, de cada propuesta, se realiza una valoración en la que se plantean pros y contras y en la que se presentan las mejores opciones por las que los planificadores se han decantado. No se incluyen presupuestos económicos ni plazos concretos de ejecución sino que simplemente se afirma que las directrices y obras que se propongan para cada puerto deberán sujetarse a la clasificación correspondiente, limitarse al cumplimiento de las condiciones mínimas exigidas en cada caso y cumplir las directrices que este Plan traza.

En cualquier caso se reconoce que este Plan y las propuestas que de él se derivan deben en todo momento ser contrastadas y corroboradas por los planes de carreteras y las propuestas de desarrollo agricola.

\section{CAMINOS Y CARRETERAS}

Si la guerra, el medio fisico y la desidia son tres constantes de la intervención española en el territorio marroquí, no cabe la menor duda que, al plantear la labor hecha en lo que respecta a los caminos y carreteras dichos tres elementos (y especialmente los dos primeros) se hacen decisivamente imprescindibles para cualquier interpretación.

Efectivamente,

"la historia del camino en Marruecos es también una historia militar. Nacen en las bases de operaciones de las campañas marroquies y van tras de las huellas que dejan el acemilero y el mulo de la Intendencia. Las bases de operaciones iniciaron luego su conversión en puertos y los caminos militares, de trazado plegado al terreno, fueron substituidos después por las actuales carreteras." (Martorell, 1946b: p. 181)

$Y$ es que si el medio fisico aporta las dificultades, durante años la guerra delimita las prioridades en el trazado y el diseño viario: quizá con la única excepción (también matizable) de la carretera Ceuta-Tetuán, construida en una de las zonas con menores problemáticas orográficas y bélicas y con una previsión de uso considerable, ${ }^{7}$ hasta la década de los años 30 la práctica totalidad de los ejes viarios del Protectorado fueron ideados con objetivos estrictamente militares, siendo a veces dificil y costosa su posterior reconversión a un uso civil (Serrano, 1936).

7 A similitud de los razonamientos planteados en el momento de construir el ferrocarril que debia unir ambas ciudades. Asl, a posteriori, esta vía es considerada "la mejor carretera, con sus cuarenta kilómetros de longitud. Su trazado tuvo en cuenta la línea de ferrocarril Ceuta-Tetuán, pero no pudo evitar el cruce con ella en algunos lugares." (Salvador, 1959: p. 183) 
Un ejemplo claro de la combinación anquilosadora de aquellos tres elementos antes citados puede ser el hecho de que hasta 1933 no se inaugura el primer tramo de la carretera (propiamente dicha y pensada ya para usos civiles) entre Tetuán y Melilla, es decir, una ruta estable y mínimamente segura entre los dos extremos de la Zona de Protectorado. El tortuoso relieve y la abrupta climatología ponían en un serio brete a los ingenieros españoles:

"A lo largo de cuatrocientos cuarenta kilómetros concurren terrenos arcillosos, roca dura, ingentes montañas y hasta llanos arenosos que exigian el empleo de una considerable suma y el esfuerzo de técnicos competentes entregados de lleno a vencer las dificultades que se amontonaban para impedir su labor." (Salvador, 1959: pp. 181182).

La estrechez de los valles, con laderas fuertemente erosionadas, y las lluvias torrenciales que ocasionalmente los llenan hacian desaconsejable su uso para el despliegue de la red de caminos rifeños de manera que en muchas ocasiones era preciso utilizar las cumbres con la subsiguiente problemática de nieblas, nieves y vientos que, entre collado y collado, añadirían una "dureza a veces excepcional" (Martínez de Velasco, 1944: p. 6) al tránsito por esta zona. Desestimado el trazado de la carretera Tetuán-Melilla por el litoral debido a la "incomodidad" generada por la sucesión de acantilados y añorando el paso de Taza (perdido en favor de la soberanía francesa en 1904) como recorrido optimo, finalmente la ruta reseguía la parte más elevada y agreste de la cordillera del Rif de manera que las diversas localidades costeras resultaban vinculadas entre sí a través de aquel eje umbilical interior o bien merced a la navegación a cabotaje.

Si bien en el primer Plan de Obras Públicas de 1940, realizado como un avance de la obra que debería proyectarse en un futuro inmediato, ya figuraba un interés explícito en predecir el trazado de futuras vías, no es hasta abril de 1944 cuando se presenta un Plan de Caminos en el marco del Plan General de Revalorización y Obras Públicas de la Zona de Protectorado. Este Plan de Caminos, redactado por el ingeniero militar Román Martínez de Velasco, resulta ser un documento de notable interés que huye de la retórica y los triunfalismos y, de una manera pragmática y sintética, afronta propuestas efectivamente implementables. Cuando se termina este Plan de Caminos ya han sido presentados los planes sectoriales referidos a Obras Hidráulicas y Portuarias; a pesar de que en buena lógica hubiera sido mejor finalizar antes el plan de ferrocarriles, las circunstancias propias del conflicto bélico mundial hicieron que pareciera más oportuno proceder antes con el de carreteras ya que el de ferrocarriles exigía unos compromisos internacionales que otras potencias (en especial Francia) no estaban en condiciones de asumir (Martorell, 1953b: p. 11).

En el primer capítulo de la primera parte del Plan se plantean y valoran los elementos que condicionan (en positivo y en negativo) los caminos y carreteras de la Zona del Protectorado. ${ }^{8}$ A diferencia de otros trabajos similares, estos aspectos no son motivo de pro-

8 Estas "condiciones" son la situación geográfica de Marruecos en las comunicaciones internacionales, la orografia y la hidrografia, la guerra, el turismo y las peregrinaciones, la irradiación de los puertos, los ferrocarriles, los núcleos de población, la población rural, la agricultura, la ganadería, la silvicultura, y la mineria. (Martínez de Velasco, 1944: pp. 3-16) 
fundo análisis (no generan una extenso estudio previo al Plan al estilo de las monografías regionales) sino que se dan por ya conocidos y ya tratados en otras ocasiones, favoreciendo una presentación rápida y utilitaria (aunque a veces rayando la plena subjetividad) tanto de los factores determinantes como de los elementos a los que debe atender una red viaria moderna.

A pesar de que algunos de aquellos condicionantes tienen un peso considerable (la herencia de la guerra, la complejidad del relieve) el autor del Plan parte no obstante con la ventaja de que prácticamente todo está por hacer. Así pues, en los capítulos siguientes plantea el establecimiento de una jerarquía viaria con su correspondiente nomenclatura y dimensiones, todo ello adaptado del Plan Español de Obras Públicas y trasladado a las caracteristicas e idiosincrasia del territorio marroqui. ${ }^{9}$ A partir de ello, se diseña el Plan de Caminos, propiamente dicho, que incluye una detallada descripción de cada una de las carreteras de la Zona (las existentes y las propuestas), su denominación, su kilometraje ${ }^{10}$ y observaciones en cuanto a las necesidades que satisface," asi como los mercados y puertos que están (o estarán) servidos por dichas carreteras. ${ }^{12}$ Una vez dibujado el futuro mapa de las carreteras de la Zona, el autor "confirma la sensatez del Plan" (según sus mismas palabras) a través de una imposible y extemporánea comparación con el caso español. La candidez de dicha validación queda quizá sobreseída por la segunda parte del Plan en el que apelando a las circunstancias excepcionales del momento presente, se expone una relación (debidamente justificada y cuantificada económicamente) de las obras, ya sean de nueva construcción o bien de reparación y acondicionamiento, que deberían realizarse en una primera e inmediata etapa.

El pragmatismo del Plan se demuestra en la selección y justificación de las obras a realizar en esta primera etapa; ${ }^{33}$ las propuestas, extremadamente realistas, hacen referencia a la necesidad de reparar puentes que son (según expresiones textuales) "peligrosisimos" o largamente "provisionales", dar comunicación a localidades y estaciones totalmente aisladas, o de acondicionar tramos actuales "inadmisibles en curvas y pendientes". En ocasiones, cuando se es consciente de que la solución ideal no será la adoptada, se proponen alternati-

9 Si el Plan Español de Obras Públicas establece tres clases de carreleras que llama nacionales, comarcales y locales de 8'00,6’50 y 5'00 metros de anchura de firme, respectivamente, admitiendo en cada una de ellas velocidades de 60, 45 y $30 \mathrm{kms} / \mathrm{h}$., el Plan de Caminos marroqul prevee caminos imperiales, comarcales, locales y elementales (con anchos de firme de 8’00,6’50, 5’00 y 4'00 m. y velocidades medias de $75,60,45$ y 30 $\mathrm{kms} / \mathrm{h}$.); aqui no se proponen autovias y no hay nada preceptivo en cuanto se refiere a los firmes pero si en lo que respecta a radio mínimo de las curvas y pendientes máximas. (Martinez de Velasco, 1944: pp. 16-18)

10 Los kilómetros de carretera existentes (y su estado) y los por construir. Una vez completado el Plan, la Zona estaria dotada de 394 kms. de Camino Imperial (Y), 1217 kms de Camino Comarcal (C), 1092 kms. de Camino Local (L), y $1159 \mathrm{kms}$ de Camino Elemental (L). (Martínez de Velasco, 1944: p. 21)

11 Se dice que pueden ser estratégicas, turisticas, portuarias, comerciales, agricolas, forestales, u otras varias. (Martínez de Velasco, 1944: anexo)

12 Cabe destacar los cinco Caminos Imperiales propuestos: Y-I de Tetuain a Ceuta, Y-II de Tetuán a Rabat, Y-III de Tetuán a Fez, Y-IV circunvalación de Tetuán, e Y-V de Melilla a Uxda. (Martínez de Velasco, 1944: anexo)

13 El importe aproximado de lo que se propone para esta primera parte supone alrededor del $25 \%$ del total, concretando que técnicamente su ejecución es posible en cinco años. 
vas, de manera que desde el Plan se abre ya la posibilidad de su misma perversión: "carretera Y-III: en algún punto donde el trazado sea muy caro, podrá hacerse una variante para abandonarla con el tiempo", "carretera E-1504: afirmar lo aprovechable y construir el resto", etc.

Igualmente, a pesar de que existen propuestas de carreteras "turisticas" o que respondan a las necesidades de los fieles en sus peregrinajes anuales, se es consciente de su escasa utilidad y que, en todo caso, deberían limitarse a la zona de Ceuta-Tánger-Tetuán ya que hay que ser "realistas" (se dice) y no construir más carreteras que las necesarias y menos si algunas sólo van a estar transitadas algunos días al año (Martínez de Velasco, 1944: p. 8).

Cabe destacar que paralelamente a este Plan se ensaya la creación de lo que se pretendia denominar Red General de Transportes por Carretera, u organismo encargado de planificar y gestionar conjuntamente las carreteras, las empresas transportistas, la señalización, los peones camineros, la policía motorizada de circulación, las estaciones de autobuses y los hoteles-paradores en ruta. No obstante, los mismos planificadores se interrogan acerca de la viabilidad de una estructura administrativa como esta en un país subdesarrollado como Marruecos y si ello no supondría un exceso de normativización e intervención por parte del estado; en cualquier caso, "debido a sus repercusiones territoriales" si que parece necesario actuar en el transporte colectivo de viajeros y por ello se redacta un Plan de Construcción de Estaciones de Autobuses (Martorell, 1946b: pp. 180-181).

Esencialmente, el Plan de Caminos estaba concebido para encauzar el tránsito de las dos grandes entradas comerciales y turísticas del exterior, Ceuta y Melilla, hasta el corazón y los extremos de la Zona y tomando como puntos de referencia las grandes ciudades del Protectorado: Tánger, Tetuán, Larache, Alcazarquivir así como Xauen y Villa Sanjurjo. Ante la dispersión del poblamiento "indígena" y el escaso valor añadido de su economía, el autor del Plan afirma que "es pues, esfuerzo perdido, proyectar ni construir caminos que sirvan a agrupaciones de viviendas indigenas" y que para dar servicio a algunos de los zocos "muchas veces será preferible construir un pequeño ramal" o incluso "trasladar el zoco a las proximidades de la carretera." En cualquier caso, parece claro para el autor del Plan que es indudablemente más importante resolver el tráfico transcontinental (por Ceuta, por derivación de la ruta Uda-Niger) que dar servicio a la población autóctona (Martínez de Velasco, 1944: pp. 4, 11-13).

\section{LA RED DE FERROCARRILES}

El proyecto, construcción y explotación del ferrocarril Ceuta-Tetuán ejemplifica a la perfección el desatino de la iniciativa privada, la indolencia de la administración pública y la falta absoluta de previsión y planificación a pesar de los discursos oficiales. Si bien este trazado no era el primero en abrirse en territorio marroquí (en 1860 se inauguró el corto tramo entre Tetuán y Río Martín) sí que debía convertirse en el más emblemático e, indudablemente, en uno de los potencialmente mas rentables. Ya a finales del siglo XIX existen diversas propuestas y numerosisimas intervenciones (en forma de conferencias, estudios, alegaciones, etc.) reivindicando la urgente necesidad de disponer de este ferrocarril. Tal como sucede muy a menudo en este tipo de proclamas, muchas de las razones esgrimidas 
son de matiz puramente patriótico: si es deber de España intervenir en Marruecos y hacerlo tanto para potenciar sus intereses particulares como para asegurar el progreso y protección de los marroquíes, una de las actuaciones con mayor aparato y con grandes posibilidades propagandísticas, que requiere labor de ingenierfa y de aplicación tecnológica y que puede tener implicaciones sobre otras múltiples actividades es, precisamente, la implantación de una línea de ferrocarril.

Con todo, también son muy presentes los argumentos que ven en este trazado ferroviario un rasgo imprescindible de la futura ordenación del territorio marroquí. En este sentido, esta línea se inscribe como pieza clave en la potenciación de Tetuán en tanto que núcleo destacado de la zona de influencia española y que debe encontrar en Ceuta la necesaria complementariedad de sus funciones económicas y urbanas. Igualmente este tramo resulta esencial para las comunicaciones que trascienden las fronteras de la zona de influencia española: el anteproyecto de enlace entre las redes española y argelina (presentado en 1906 al ministro de Fomento español) a través de Melilla y resiguiendo la costa mediterránea, tiene en la sección Ceuta-Tetuán el primer recorrido (Garcia Faria, 1906: pp. 54-56). Además, el interés por situar en Ceuta una terminal de ferrocarril de ámbito continental aumenta en el marco del conflicto internacional de la II Guerra Mundial: si en algún momento se propuso una línea que permitiese la "vertical ideal de penetración" a través de Ceuta, Tetuán, Xauen y hasta Fez, en este período histórico parece suficiente y ya de por si interesante la construcción de un ramal de la línea Tánger-Fez que, partiendo de Tzenin Sidi Yamani (entre Arcila y Larache), llegue hasta Ceuta (Méchin, 1928; Martorell, 1946b: pp. 179-180). En cualquier caso, el tramo Ceuta-Tetuán resulta central y de paso obligado.

Por si fuera poco, el territorio que debería atravesar la citada línea no presentaba excesivas dificultades orográficas (al menos en comparación con el problemático relieve existente en gran parte de la zona de influencia española, especialmente el Rif) de manera que la capacidad tecnológica de la ingeniería española era, aparentemente, suficiente para afrontar dicha construcción. Si bien en algunos momentos la actuación guerrillera de algunas cabilas rebeldes hizo peligrar las obras, ciertamente se trataba de una de las regiones más seguras y fácilmente protegibles.

A pesar del consenso existente en lo que se refiere a este carácter imprescindible, central y ejemplar del trazado y a pesar también de las, en principio, escasas dificultades técnicas, no existe una propuesta oficial de construcción hasta el año 1906 y no es hasta 1913 cuando el gobierno español dicta los proyectos y disposiciones preliminares que habrian de iniciar las obras. Si bien el organismo inicialmente encargado de llevar a cabo la construcción del ferrocarril era la Alta Comisaria, la falta de recursos económicos, de oportunidad politica y de visión de futuro (y, se añadirá, "y el exceso de burocracia"; Anónimo, [1918]; p. 201) detendrán el proyecto que sólo se desbloqueará cuando se anuncie un concurso abierto a la iniciativa de empresas privadas. Ante las poco entusiastas condiciones establecidas (la empresa constructora deberá adelantar el coste total del proyecto, se concederá la explotación de la línea por un período de tan sólo cinco años, etc.) al concurso de mayo de 1916 sólo accede, bajo presiones, la Compañía Española de Colonización. El proceso de contratación y, sobretodo, el de construcción, se verá plagado de conflictos: a pesar de que el ancho de vía previsto debía ser de 1'45 m. (ancho "europeo") el ejército impone el ancho de 
$1 \mathrm{~m}$. "con carácter provisional" y con la falsa esperanza de pronta transformación; la defensa militar del trazado en construcción debe afrontar graves e inesperados ataques de fuerzas rebeldes que causan numerosas bajas y un considerable retraso; la perforación de algún túnel y la construcción de ciertos viaductos para vadear pequeños ríos y arroyos inician amplios debates tecnológicos y disparan el presupuesto hasta cifras desorbitadas (Ruiz, 1987: pp. 360-361; Anónimo, [1918]: pp. 205-212). Un ejemplo de estos desvíos financieros y del pésimo trabajo de ingeniería realizado son las críticas que, incluso años después de independencia de Marruecos, todavía subsistían sobre el trazado del ferrocarril Ceuta-Tetuán. Así, en referencia al acceso a Tetuán por la carretera de Ceuta, se decía en 1962 que

"se supeditó a la construcción del túnel del ferrocarril Ceuta-Tetuán, incurriéndose, a nuestro entender, en un error de planteamiento económico, a) porque la inversión realizada en el citado túnel valía casi lo que todo el ferrocarril y b) porque para que esta inversión hubiera tenido lógica debiera haber sido acompañada de la ejecución del previsto enlace con el de Tánger, y esta obra valía ella sola más que todo el primer plan quinquenal del cual se financió el túnel." (Sierra, 1962: p. 89)

Finalmente, la línea se inaugura en 1918 con una rentabilidad económica que irá en aumento hasta 1924 cuando la pacificación, la mejora en el trazado de las carreteras y en el aprovisionamiento de carburante para los vehículos a motor llevarán al ferrocarril a un período de decadencia y crisis financiera. Las circunstancias especiales generadas por la Guerra Civil suponen una cierta revitalización de la línea que coincide con el fin de la gestión por parte de la Compañia Española de Colonización y el inicio de su explotación en manos de la administración pública del estado español (1938). A pesar de que locomotoras (muchas de ellas de fabricación norteamericana) procedentes de otras líneas cerradas se traspasan a la línea Ceuta-Tetuán, en 1942, de las catorce existentes ninguna de ellas estaba en perfecto servicio: faltan repuestos y combustible, las averias son lo más habitual y el déficit es ya crónico. La independencia de Marruecos en 1956 contribuirá a agravar algunos de dichos problemas de manera la línea será clausurada el 1 de julio de 1958 (Ruiz, 1987: pp. 361-363).

Si esta es la deplorable historia de la línea que, además de ser la más rentable y más necesaria, era considerada exponente ejemplar de la acción española, el proceso seguido por otras muchas líneas menores (de reducido kilometraje y corta vida útil, a veces vinculadas a las explotaciones forestales o mineras y muy a menudo a las necesidades del ejército: Touceda, 1960) sólo es digna de mención en tanto que sirven a los técnicos para reclamar en reiteradas ocasiones la necesidad de una planificación global y coherente y más teniendo en cuenta el carácter vertebrador del territorio que se atribuye al ferrocarril y el papel central que este pareceria tener en el contexto de las comunicaciones euro-africanas (Martorell, 1943: pp. 7-18).

Así, el Plan de Ferrocarriles ${ }^{14}$ es, de hecho, un intento de racionalizar la red existente añadiendo diversas nuevas rutas que contribuyan a ello y de sistematizar, a nivel técnico y de gestión, todo lo referente a los ferrocarriles marroquíes. El Plan incide, de entrada, en la

14 Integrado en el marco de los trabajos de planificación territorial y sectorial para Marruecos de los años 40 (Alta Comisaría de España en Marruecos, 1948: pp. 535-573). 
necesidad de "unificar tanto el material tractor como el móvil" pero, sobre todo, regularizar el ancho de vía que debería coincidir con el "internacional", como única fórmula para asegurar las conexiones con el resto del continente africano y potenciar así el papel de Ceuta como punto terminal continental. El objetivo es igualmente evitar nuevos errores y soluciones provisionales que a la larga aumentan los costes de manera espectacular. ${ }^{15}$

En la segunda parte del Plan se presentan todos los trazados propuestos y realizables, acompañados de algunas consideraciones encaminadas a esbozar la rentabilidad probable de las líneas y los perfiles longitudinales de los trazados. Así pues, además de adecuar las líneas en servicio (Tánger-zona francesa vía Alcazarquivir; Larache-Alcazarquivir; CeutaTetuán' ${ }^{19}$ ) se proponen las nuevas líneas Ceuta-frontera tangerina, Tetuán-Larache y TetuánMelilla (vía Xauen y Villa Sanjurjo) con sus ramales Xauen-Alcazarquivir, Xauen-zona francesa hacia Fez, asi como Zeluán-zona francesa hacia Uxda, Monte Arruit-zona francesa hacia Guercif.

A pesar de que se reconoce que sólo la ruta Ceuta-Tetuán-Larache-Alcazarquivir será rentable económicamente, la construcción del resto de las líneas responde a una imprescindible "necesidad social" (posibilitar las conexiones internacionales, potenciar las zonas de nueva colonización agrícola, etc.). A pesar de tener presentes en la memoria las grandes dificultades observadas en la adjudicación y mantenimiento de la línea Ceuta-Tetuán, y "para evitar grandes desembolsos al Estado" el Plan prevee que la construcción y la gestión de estas líneas recaiga en empresas privadas que ofrezcan un "servicio al público" bajo la supervisión estatal.

\section{EL PLAN DE REVALORIZACIÓN ECONÓMICA DE LA ZONA}

Los responsables de este Plan ${ }^{17}$ reconocen, en sus mismos prolegómenos, que se trata casi de un apéndice, de un complemento de los aspectos señalados en el Plan de Obras

15 Además de otros casos ya mencionados, el Plan recuerda "un ejemplo clásico", el del túnel del Rincón "construido provisionalmente (parece ser que por razones económicas), con idea de sustituirlo más adelante y que todavia sigue en funcionamiento. Las grandes pendientes que forman sus accesos obligan a reducir los tonelajes de los trenes en un 40 por 100 aproximadamente; por lo que son precisos trenes de mercancias complementarios, por lo menos cuatro dias a la semana. Lo que en combustibles y grasas para las máquinas representa unas 15.000 pesetas mensuales. Si incluyéramos el gasto de personal, que es preciso reforzar en los equipos de máquina y tren, y en vias y obras, para conservar esas fuertes pendientes, tendremos las 200.000 pesetas que anualmente incrementan los gastos de explotación de la linea. Suponiendo un interés del 6 por 100 , zes que el túnel "definitivo" hubiese costado 3.300 .000 pesetas más que el construido? Y no hemos evaluado lo que disminuye la capacidad de competencia del ferrocarril con la carretera a causa del mayor tiempo invertido." (Alta Comisaria de España en Marruecos, 1948: pp. 541-542)

I6 En la línea Ceuta-Tetuán "el estado de agotamiento del material, la acción demoledora de los agentes atmosféricos sobre las obras de tierra y la superestructura y, finalmente, el gálibo del túnel de Rincón obligan a un estudio del ferrocarril en el que casi puede asegurarse que no se aprovecha nada de los existente" (Alta Comisaria de España en Marruecos, 1948: p. 558).

17 Cuyo nombre completo es el de Plan de Revalorización Económica de la Zona en Agricultura y Ganadería, Montes, Minas e Industrias. Sobre la explotación y transformación de los recursos naturales de la Zona (agricultura, ganadería, mineria, pesca, industria) véase el capitulo 15 de esta misma obra. 
Públicas. Asi, a pesar de que el Plan de Obras Hidráulicas incluye numerosas acciones destinadas a incrementar la extensión del área cultivable y de la productividad agricola, una parte destacada del Plan de Revalorización Económica de la Zona se dedica a la agricultura. En él, además de los habituales estudios previos sobre la situación presente de la estructura agraria, se indican acciones cuyo objetivo genérico es

"pasar de una agricultura primitiva y rutinaria a otra más progresiva [...] por lo que el plan agricola está trazado bajo un aspecto integral, que aborda la capacitación e instrucción de los agricultores, la selección y producción de semillas y ganados, el suministro de plantones, etc., y una serie de obras de tipo económico y social como son las transformaciones de secano en regadío, la puesta en cultivo de terrenos y constitución de colonias, las plantaciones de arbolado en extensas superficies, etc. y atendiendo tanto al aspecto económico de la reforma, como al social." (PASTOR, 1946: pp. 89-90)

El Plan prevee actuaciones "de repercusión indirecta y general sobre el pais" (granjas, viveros, etc.) y obras "de repercusión directa y más intensiva sobre zonas limitadas" (regadios, colonias, plantaciones, etc.), atendiendo a las limitaciones impuestas por el clima, la meteorología, la topografia y el tipo de suelo. Las consideraciones técnicas son detalladas y perfectamente adecuadas a las características y necesidades de la Zona. A la ganadería, que se estima complementaria de la agricultura, también se le dedican extensos apartados en los que se reconoce igualmente el largo trecho todavia por recorrer hasta conseguir unas explotaciones modernas y rentables. ${ }^{18}$

El modelo óptimo a seguir es el del establecimiento de colonias agrícolas integradas, en las que se conceden viviendas y lotes de tierra a los colonos (de unas $20 \mathrm{ha}$.) con unas condiciones de créditos y amortización preferentes.

La revalorización forestal se justifica ante la constatación de que, debido a lo agreste del relieve rifeño, dos terceras partes de la superficie de la Zona sólo son susceptibles de aprovechamientos forestales. En este caso, el Plan se reduce a proclamar unos criterios de vigilancia, defensa, regeneración y repoblación de las masas forestales, y a establecer las pautas básicas en la explotación maderera y corchera (instalaciones, concesiones, cupos, etc.). ${ }^{19}$

En lo que respecta a la revalorización minera, el Plan se limita a señalar las explotaciones existentes y a reafirmar la necesidad de reserva de determinadas áreas para prospecciones y eventuales explotaciones futuras (posibilidad de petróleo, pizarras bituminosas, grafito, plomo, etc.). A pesar de que es el Majzen quien otorga las concesiones a través de la administración española (mayormente el Instituto Nacional de Industria) el Plan no deja lugar a dudas de que es la iniciativa privada la que llevará a cabo cualquier explotación minera, tal como ha sido habitual desde finales del siglo XIX. ${ }^{20}$

18 Véase también Junta Central de Colonización y Repoblación Interior (1928), Aranda (1942), Melis (1950), Chaara (1990) y Rivero (1994).

19 Véase también Vázquez del Rio (1935), Anónimo (1941), Sánchez (1942) y Pavón (1957).

20 Véase también Pastora (1932) y Rivet (1979). 
En relación al apartado referente a la revalorización industrial, y tal y como ya se intuía en el caso de las explotaciones mineras, no puede hablarse de planificación desde la administración pública ya que se deja a la libre iniciativa privada la decisión de la creación y transformación de fábricas y empresas de todo tipo. ${ }^{21}$ De hecho sólo se pretende "estimular y encauzar" las nuevas industrias y, para hacerlo, se menciona la creación de la Inspección de Industrias (que debería establecer una estadística industrial, velar por el recto cumplimiento de las obligaciones de las empresas concesionarias de servicios públicos, proteger las patentes y marcas, establecer normas técnicas, etc.) paralelamente a otros organismos que se estiman complementarios, tales como el Servicio de Meteorología y el de Estadística. ${ }^{22}$

\section{LA INVENCIÓN DE UNA POLÍTICA TERRITORIAL: PLANES TERRITORIALES Y PLANES QUINQUENALES}

Si bien a principios de siglo ya existen algunas propuestas sistemáticas (algunos "planes") de intervención en Marruecos, ${ }^{23}$ tan sólo el período comprendido entre 1939 y 1956 puede considerarse como "normal" a efectos de una política colonizadora plena, a diferencia de lo que ocurre en el Protectorado francés de Marruecos donde la eficacia presidió una actuación que, por otra parte, se desarrollaba en un contexto mucho más favorable desde el punto de vista de los recursos disponibles como de la actitud de la población.

Así pues, puede afirmarse que la planificación territorial marroquí se inicia en 1940 con la redacción de un sucinto Plan de Obras Públicas que, de hecho, sirvió de pauta para todos los documentos posteriores ya más estructurados. El Alto Comisario general Luis Orgaz, sería el impulsor de un equipo de técnicos que, dirigidos y animados por Vicente Martorell en tanto que Delegado de Obras Públicas, se encargó de redactar las distintas partes de un extenso Plan de Obras Públicas y Agrícolas ${ }^{24}$ que

"si en sus detalles habrá de sufrir variaciones, creo que durante muchos años habrá de servir de directriz a los técnicos a quienes se encomiende la misión de incrementar el desarrollo económico del pais." (Gudín, 1962: p. 104)

21 La descripción detallada de las industrias existentes en la Zona demuestra que, básicamente, se trata de empresas artesanales, relacionadas con la transformación de productos agrarios y minerales y, en su gran mayoria, de una dimensión estrictamente local (Alta Comisaria de España en Marruecos, 1948: pp. 419-489). Véase también Touceda (1950b).

22 El Plan concede muy escasa importancia a la pesca (véase Alta Comisaria de España en Marruecos, 1948: pp. 375-394; véase también Touceda, 1950a).

23 En 1908 el Ministro de Fomento J. Garcia Prieto, formuló un plan de ordenación que, en síntesis, abarcaba los elementos esenciales de las infraestructuras y los equipamientos necesarios para Marruecos (puertos, ferrocarriles, carreteras, zocos, elc.; Gómez-Jordana, 1976: p. 22). En 1927 se crea la Dirección General de Colonización, que habia de dividirse en las siguientes secciones: propiedad territorial, colonización, agricultura y ganadería, montes, comercio, y economia rural; con el advenimiento de la II República este organismo se suprime convirtiéndose en un negociado de la Delegación de Fomento (Gómez-Jordana, 1976: pp. 239-240).

24 Que contenia también una propuesta de red de carreteras pero no incluía las obras hidráulicas dado que "por su mayor estudio y conocimientos, no era posible improvisar" (Lambea, 1942a: p. 1). 
El mismo general Orgaz fue el que ordenó

"la formación de un Plan Decenal (1946-56) que integrado en el Plan General de Revalorización y Obras Públicas recogiese la obra inmediata y la valorase, para acudir al gobierno español en demanda de un compromiso de asistencia anual durante un decenio. El primer Plan Decenal [...] está redactado en forma de sucinta memoria en la que, tras un ligero preámbulo, se justifica el pensamiento que inspiró la determinación de las obras dentro de cada uno de los siguientes epigrafes: Plan de Caminos. Plan de Obras Hidráulicas, Plan de Ordenación Portuaria, Plan de Transportes Terrestres, Plan Agronómico, Plan Forestal, Plan de Ordenación Urbana." (Martorell, 1946b: p. 187)

La documentación correspondiente a este Plan Decenal y al conjunto de planes sectoriales parciales es la que recoge el libro Acción de España en Marruecos. La obra material. ${ }^{25}$ En la práctica este Plan Decenal es, simplemente, la consideración conjunta de los citados planes sectoriales (redactados entre 1942 y 1945) sin ninguna visión conjunta o plan territorial global. Además, en Acción de España en Marruecos dicha secuenciación decenal queda traspasada a unos "Planes Quinquenales" (teóricamente unos diez) que, de hecho, vienen exclusivamente delimitados por el periodo de amortización de los empréstitos obtenidos. ${ }^{26}$ Sólo en alguno de los planes sectoriales existe una secuenciación de las obras propuestas y no siempre coinciden con quinquenios; no hay análisis de viabilidad financiera de las propuestas; no existen valoraciones económicas y sociales que secuencien o prioricen las intervenciones en su conjunto; no queda claro el papel de la administración pública en la planificación y en la gestión del territorio.

El contenido y los objetivos del Plan Decenal (o, de hecho, del Plan Quinquenal) son a menudo confusos: ${ }^{27}$ quizá la falta de una tradición de ordenación territorial se haga aquí más patente que en la redacción de los planes sectoriales ya que de lo que ahora se trataba era de establecer criterios básicos de desarrollo, prioridades de intervención, apuestas a largo plazo. Se intuye, pero no se consigue, establecer una política territorial, algo todavía inédito en España. En cualquier caso sorprende la terminologia usada (los planes "quinquenales" tienen muchas reminiscencias soviéticas) y la precocidad (en España se empezará a hablar de Planes de Desarrollo, cuatrienales, en 1959), lo que viene a corroborar, una vez más, que se trata de una actuación propagandística del régimen de Franco para evidenciar el singular interés y compromiso del Estado Español en relación a Marruecos.

25 Publicado en 1948 por la Alta Comisaria de España en Marruecos bajo la dirección de Vicente Martorell. Véase el anålisis hecho en Albet et alii (1995).

26 El primer empréstito, de 260 millones de pesetas, fue aprobado en 1946 y debia distribuirse (y ejecutarse las obras correspondientes) en el periodo de las cinco anualidades siguientes lo que, de implementarse correctamente, debería generar un ritmo "quinquenal"de actuaciones. Una vez más, cabe remarcar tanto el procedimiento (un empréstito) como el monto de la acción, totalmente insólitos para la economía depauperada y autárquica de la España de la época.

27 Véase especialmente las páginas 9-15 y 791-800 de Alta Comisaria de España en Marruecos (1948). 
A pesar de las buenas intenciones depositadas en los planes, en 1953 (sólo tres años antes de la independencia), Vicente Martorell, coordinador del Plan Territorial que resulta ser la obra Acción de España en Marruecos, reconoce que "su" plan territorial

"es un documento escasamente consultado y que todavia no ha permitido alcanzar el tan deseado Plan de Ordenación Económica de la Zona de Protectorado de España en Marruecos" (Martorell, 1953b: p. 4).

No obstante este fracaso en su implementación, hay que tener muy presente el notable interés de sus contenidos y la valoración global extremadamente positiva de esta obra de sintesis. En algunos capítulos o párrafos se retoman los textos originales de cada uno de los planes sectoriales considerados mientras que en otros se presenta un resumen; ello confiere una gran diversidad a esta obra a la vez que una considerable heterogeneidad a los criterios de estudio y de presentación de los contenidos así como el lenguaje usado. A pesar de ello, el conjunto presenta todos los elementos propios de un trabajo técnico de ordenación territorial junto con ciertos rasgos propios de las monografias regionales.

La estructura interna de la obra es muy coherente: siendo conscientes de que el primer factor que debe asegurarse es el de la subsistencia, deben priorizarse los trabajos hidráulicos y racionalizarse la gestión de los recursos naturales (especialmente las explotaciones forestales y ganaderas). Dado que se prevee que este énfasis no ofrecerá suficientes resultados tangibles, hará falta asegurar las importaciones desde el exterior (de ahí la importancia de los puertos, los ferrocarriles y las carreteras; Martínez de Velasco, 1944: pp. 8-9; Martorell, 1946b: p. 174). Una vez asegurada la subsistencia habrá que velar por la salud de la población, lo que implica la construcción de viviendas dignas; la salud "espiritual" llegará gracias a la educación (con la subsiguiente edificación de escuelas y la política de escolarización) mientras que la renovación de la administración pública exigirá la instalación de nuevos equipamientos oficiales. Finalmente, atendiendo a la historia y la cultura autóctonas, la planificación deberá tener también en cuenta la transmisión del patrimonio artístico y monumental.

\section{BIBLIOGRAFÍA}

ALBET i MAS, Abel; GARCIA RAMON, M. Dolors; NOGUÉ i FONT, Joan \& RIUDOR i GORGAS, Lluís (1995), "Géographie, aménagement du territoire et colonialisme espagnol au Maroc", Cahiers de Géographie de Québec, 39 (106): pp. 43-59.

ALTA COMISARIA DE ESPAÑA EN MARRUECOS (1946), Labor de España en Africa, Barcelona: Alta Comisaria de España en Marruecos.

ALTA COMISARÍA DE ESPAÑA EN MARRUECOS (1948), Acción de España en Marruecos. La obra material, Tetuán \& Madrid: Alta Comisaría de España en Marruecos (Talleres del Instituto Geográfico y Catastral).

ANÓNIMO (1918), Ceuta-Tetuán Tetuán: Editorial Hispano Africana.

ANÓNIMO (1941), "Los montes en Marruecos", Mauritania, 167: pp. 303-306. 
ARANDA MATA, Antonio (1942), "Presente y porvenir de Marruecos", Africa. Revista Española de Colonización, 1: pp. 3-9.

CÁMARA URZÁIZ, Juan de la (1932), La conquista del Muluya. Aprovechamiento integral de las aguas de este río, Melilla: Papelería e Imprenta "La Española".

CHAARA, Ahmed (1990), Paysans, pêcheurs et migrants. Les campagnes des littoraux du Rif Occidental (Thèse de Doctorat en Géographie; Université de Lille).

FONT DE MORA, Rafael (1936) "Cielo y tierra", La Gaceta de Africa (núm. extraordinario dedicado a las ciudades de Marruecos); pp. 101-102.

GALLEGO ARANDA, Salvador (1996), Enrique Nieto en Melilla: la ciudad proyectada, Granada: Universidad de Granada \& Centro Asociado de la UNED de Melilla.

GARCÍA FARIA, Pedro (1906), Cuatro palabras acerca de Marruecos y sus futuros ferrocarriles, Madrid: Establecimiento Tipográfico de los Hijos de J.A.Garcia.

GARCÍA FIGUERAS, Tomás (1939), Marruecos (La acción de España en el Norte de Africa), Barcelona: Ediciones Fe.

GÓMEZ-JORDANA SOUZA, Francisco (et alii) (1976), La tramoya de nuestra actuación en Marruecos, Madrid: Editora Nacional.

GONZÁLEZ QUIJANO, Pedro M. (1942), "La política hidráulica en Marruecos", Boletin de la Real Sociedad Geográfica, 78; pp. 402-429.

GUDÍN FERNÁNDEZ, Tomás (1962), "Las obras hidráulicas en Marruecos", Archivos del Instituto de Estudios Africanos, 64; pp. 101-112.

JUNTA CENTRAL DE COLONIZACIÓN Y REPOBLACIÓN INTERIOR (1923), Estudios e informes relativos a la colonización agricola de la Zona de protectorado de España en Marruecos, Madrid: JCCRI.

LAMBEA PALACIOS, Daniel (1942a), Anteproyecto de Plan de Obras Hidráulicas en el Protectorado Español de Marruecos, Tetuán: Alta Comisaría de España en Marruecos: Delegación de Obras Públicas y Comunicaciones.

LAMBEA PALACIOS, Daniel (1942b), Las obras hidráulicas en el Protectorado Español de Marruecos, Tetuán: Alta Comisaria de España en Marruecos.

LLONCH GURREA, José Antonio (1992), La arquitectura popular religiosa en el norle de Marruecos: Tetuán, Barcelona: Servei de Publicacions de la Universitat Politècnica de Catalunya.

MARTÍNEZ DE VELASCO, Roman (1944), Plan General de Revalorización y Obras Públicas de la Zona. Plan de Caminos, Tetuán: Alta Comisaría de España en Marruecos (Delegación de Obras Públicas y Comunicaciones).

MARTORELL OTZET, Vicente (1943), Los ferrocarriles en nuestra Zona de Protectorado, Tetuán: Alta Comisaría de España en Marruecos.

MARTORELL OTZET, Vicente (1946a), "El plan de obras públicas en la zona de Protectorado", Africa. Revisła Española de Colonización, marzo. 
MARTORELL OTZET, Vicente (1946b), "Las obras públicas en la puesta en valor de nuestra Zona de Protectorado", in ALTA COMISARÍA DE ESPAÑA EN MARRUECOS Labor de España en Africa, Barcelona: Alta Comisaría de España en Marruecos; pp. 169-188.

MARTORELL OTZET, Vicente (1952), "El segundo plan quinquenal", Africa. Revista Española de Colonización, 129-130; septiembre octubre.

MARTORELL OTZET, Vicente (1953a) "El Zaio. Un poblado de 500 habitantes que llegará a 12.000 dentro de veinticinco años", Africa. Revista Española de Colonización, 133; pp. 6-10.

MARTORELL OTZET, Vicente (1953b), Las obras puiblicas en Marruecos. Diez años de experiencia proyectados sobre el futuro, Tetuán: Alta Comisaría de España en Marruecos.

MÉCHIN, Réné (1928), Les chemins de fer au Maroc. Paris: Presses Universitaires de France.

MELENCHÓN GARCIAA, Juan; PINEDO REYES, Pablo \& SÁNCHEZ MARFIL, José Antonio (s.d.), Tetuán, entre el Dersa y el Gorgues, Tetuán: ITE "Juan de la Cierva".

MELIS CLAVERÍA, Manuel (1950), "Africa española: su economía", in DIRECCIÓN GENERAL DE MARRUECOS Y COLONIAS, Curso de divulgación africanisła en la Universidad de Valladolid, Madrid: Instituto de Estudios Africanos-CSIC; pp. 31-51.

OCHOA Y BENJUMEA, José (1944), "Los puertos de Marruecos y posesiones españolas de Africa del Norte", Boletín de la Real Sociedad Geográfica, 80; pp. 331-355.

PASTOR MORENO, José Maria (1946), "El Plan de Revalorización Económica de la Zona en Agricultura y ganadería, Montes, Minas e Industrias", in ALTA COMISARÍA DE ESPAÑA EN MARRUECOS, Labor de España en Africa, Barcelona: Alta Comisaría de España en Marruecos; pp. 85-104.

PASTORA, José Luis (1932), La minería en la Zona Española del Protectorado de Marruecos, Madrid: Sucesor de Enrique Teodoro.

PAVÓN, M. (1957), La repoblación forestal en la práctica, Larache: Artes Gráficas Boscá. PLAZA NAVARRO, Manuel de la (1936), "Las ciudades del Protectorado", La Gaceta de Africa (núm. extraordinario dedicado a las ciudades de Marruecos); p. 1.

RIVERO CORREDERA, J. (1994), La colonisation agraire dans le Rif Oriental d'hier à aujourd'hui, Oujda (Colloque).

RIVET, D. (1979), "Mines et politique au Maroc. 1907-1914", RHMC, 26: pp. 559-578.

ROMÁN, Juan (1994), Fragmentos de una conversación continua sobre Alhucemas, Melilla: Ayuntamiento de Melilla.

RUIZ PELÁEZ, Joaquín (1987), "Comunicaciones entre ambas orillas del estrecho de Gibraltar. Importancia de la línea férrea Ceuta-Tetuán", in Actas del Congreso Internacional: El Esirecho de Gibraltar. vol. $I V$, Madrid: UNED-Ayuntamiento de Ceuta; pp. 357-373. 
SALVADOR, Manuel (1959), "España y sus carreteras en Marruecos", Mauritania, 378; pp. 180-184.

SÁNCHEZ COZAR, Santiago (1942), "Explotaciones forestales en el Protectorado", in Cómo es la Zona del Protectorado Español en Marruecos, Barcelona: SIEM; pp. 7194.

SERRANO PIÑANA, Juan (1936), "La red de comunicación [sic] secundarias de la zona", La Gaceta de Africa (núm. extraordinario dedicado a las ciudades de Marruecos); pp. 4-5.

SIERRA OCHOA, Alfonso de (1962), "Urbanismo y vivienda en Tetuán", Archivos del Instituto de Estudios Africanos, 64; pp. 75-100.

TOUCEDA FONTENLA, R. (1950a), "La ballenera de Bensư”, Mauritania, 276; p. 252.

TOUCEDA FONTENLA, R. (1950b), "La revalorización de España en Africa", Mauritania, 275; p. 231.

TOUCEDA FONTENLA, R. (1960), "Los ferrocarriles en Marruecos", Mauritania, 397; pp. 426-430.

VÁZQUEZ DEL RÍO, Manuel (1935), Arbolado y bosques en Marruecos, Ceuta: Alta Comisaría de España en Marruecos. 